\title{
Voltage Mode Pulse Width Modulator Using Single Operational Transresistance Amplifier
}

\author{
Rajeshwari Pandey, ${ }^{1}$ Neeta Pandey, ${ }^{1}$ and Sajal K. Paul ${ }^{2}$ \\ ${ }^{1}$ Department of Electronics and Communication, Delhi Technological University, Bawana Road, Delhi 110042, India \\ ${ }^{2}$ Department of Electronics Engineering, Indian School of Mines, Dhanbad, Jharkhand 826004, India \\ Correspondence should be addressed to Neeta Pandey; n66pandey@rediffmail.com
}

Received 31 August 2012; Revised 2 December 2012; Accepted 10 December 2012

Academic Editor: Kyoung Kwan Ahn

Copyright ( 2013 Rajeshwari Pandey et al. This is an open access article distributed under the Creative Commons Attribution License, which permits unrestricted use, distribution, and reproduction in any medium, provided the original work is properly cited.

This paper presents a voltage mode pulse width modulator (PWM) using single operational transresistance amplifier (OTRA). The proposed PWM consists of a relaxation oscillator output which is modulated using modulating signal. PSPICE simulation results and experimental results have been included to verify the theoretical analysis.

\section{Introduction}

Pulse width modulation (PWM) scheme is widely used in communication systems, DC motor speed controller, power conversion control circuits [1-3], and instrumentation. In PWM, the width of pulses of a pulse train is changed in accordance to voltage level of modulating signal. The PWM generators are available in Integrated Circuits (ICs) form; however, the internal circuitry is somewhat complex and typically consists of current sources, flip-flop, comparators, and analog switches [4]. Apart from these readily available ICs, the PWM signal can most commonly be generated by comparing a modulating signal with a sawtooth or triangular waveform. Alternatively, information signal can first be combined with a sawtooth or triangular waveform and thereafter comparing the combined signal with a reference level to generate PWM signal. This can be implemented using a Schmitt trigger with an RC circuit in feedback loop [5]. Extensive literature review reveals that PWMs based on this concept are available using different active analog building blocks such as op-amps [6], current conveyor II (CCII) [4], and operational transconductance amplifier (OTA) [7, 8]. High-frequency performance of PWMs employing opamps is limited due to lower slew rate and constant gainbandwidth product of the op-amps. The CCII-based PWM has the advantage of generating accurate PWM signal with high operating frequencies [4], and the output amplitude and frequency of the PWMs based on OTAs can be independently/electronically tuned [7]. Despite these advantages, the circuits proposed in $[4,7]$ suffer a drawback of using excessive number of active elements. Reference [8] presents another OTA-based PWM which uses derivative method. In derivative method, the duty cycle of PWM signal depends on a differentiated result of the modulating signal. This method is not suitable for applications where the changes in modulating signal are rare, such as in power converters [7]. The open literature suggests that a PWM pulse train can also be produced with the use of voltage-controlled delay lines [9], current-controlled delay cells [10], and voltage-controlled phase shifter [11] wherein the underlying concept is not the same as used in [4-8]; that is, the comparison of modulating signal with reference triangular/sawtooth waveform [9] uses two voltage-controlled delay lines, a fixed delay element, two rising edge detectors, along with an RS latch to generate a $0 \%-50 \%$ duty cycle at the output of the latch. Two $0 \%-50 \%$ modulators are connected in parallel to extend the duty cycle to $100 \%$. Current-controlled delay cell-based duty oscillator and pseudohyperbola charge current generator are used in [10] for generating PWM. Yet, another PWM is proposed in [11] which employs a voltage-controlled phase shifter, a twoinput logic AND gate, a network of logic inverters, and FET 
switches. This circuit generates a $0 \%$ to $50 \%$ pulse width using the phase shifter and an AND gate and then extends the range to $0 \%-100 \%$ using the inverters.

This paper aims at presenting an operational transresistance amplifier (OTRA) based PWM, using a relatively simpler scheme, wherein the modulating signal is combined with an exponential carrier waveform and compared with a reference. This paper is organized as follows. In Section 2, the function of an OTRA is briefly described, followed by the operation principle of the proposed PWM circuit. The feasibility of the presented circuits is verified through circuit simulations and experimental results in Section 3. It is observed that the simulation and experimental results are in close agreements with theoretical propositions. The comparison of the proposed circuit with earlier reported structures is presented in Section 4. Section 5 concludes the paper.

\section{Circuit Descriptions}

OTRA is a high gain current input voltage output device. The input terminals of OTRA are internally grounded, thereby eliminating response limitations due to parasitic capacitances and resistances and, hence, are appropriate for high-frequency operation. The circuit symbol of OTRA is shown in Figure 1, and the port characteristics are given by (1), where $R_{m}$ is transresistance gain of OTRA. For ideal operations, the $R_{m}$ of OTRA approaches infinity and forces the input currents to be equal. Thus, OTRA must be used in a negative feedback configuration. Consider

$$
\left[\begin{array}{l}
V_{p} \\
V_{n} \\
V_{o}
\end{array}\right]=\left[\begin{array}{rrr}
0 & 0 & 0 \\
0 & 0 & 0 \\
R_{m} & -R_{m} & 0
\end{array}\right]\left[\begin{array}{l}
I_{p} \\
I_{n} \\
I_{o}
\end{array}\right] .
$$

The general scheme of PWM is depicted in Figure 2. The modulating signal and carrier signal are first summed up, and then PWM output signal is generated by comparing the summation signal and reference level.

The proposed PWM circuit is shown in Figure 3. The circuit consisting of OTRA, resistors $R_{F}$ and $R_{L}$, and capacitor $\mathrm{C}$ serves as square wave generator. Exponential voltage waveform across capacitor $\mathrm{C}$ serves as carrier waveform. The modulating signal $v_{i}$ and the carrier wave are summed up at node $p$ through resistor $R_{s}$. Thus, the voltage across the capacitor will be summation of carrier and modulating signal, and the PWM output is available at $v_{o}$.

The resistor $R_{F}$ and capacitor $\mathrm{C}$ form a positive feedback loop. $V_{\text {sat }}^{+}$and $V_{\text {sat }}^{-}$are the two possible saturation levels of output $v_{0}$. Assume initially that $v_{0}$ switches to saturation level $V_{\text {sat }}^{+}$at $t=0$, as shown in Figure 4 . This results in charging of the capacitor present in the feedback loop, and the voltage across the capacitor reaches $V_{\mathrm{TH}}$, a value at which $I_{p}$, the current through $p$ terminal, becomes slightly less than the current through $n$ terminal, $I_{n}$. As a result, the output voltage switches to $V_{\text {sat }}^{-}$, and the capacitor starts charging in the opposite direction. Now, as capacitor voltage approaches $V_{\mathrm{TL}}$, the output once again switches back to $V_{\text {sat }}^{+}$.

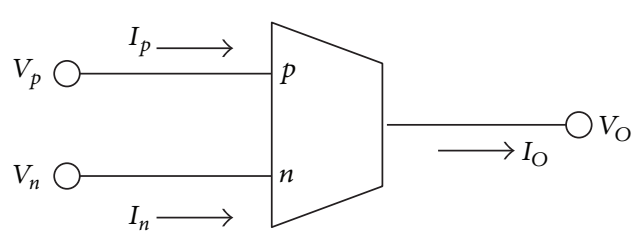

FIGURE 1: OTRA circuit symbol.

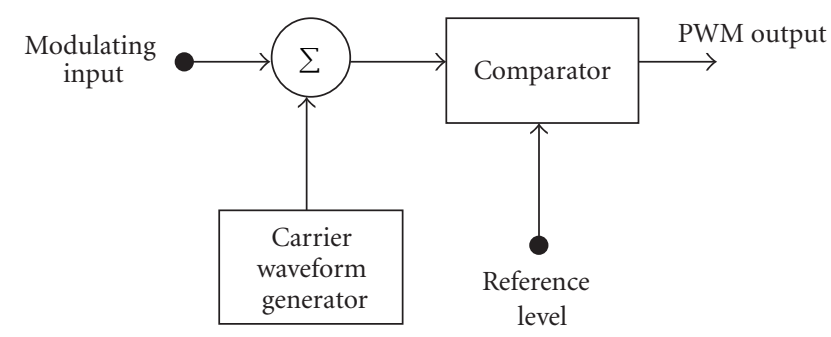

FIGURE 2: The scheme of PWM [4].

The $V_{\mathrm{TH}}$ and $V_{\mathrm{TL}}$ values can be obtained from the routine analysis of this PWM circuit and are expressed by (2) and (3), respectively. Consider

$$
\begin{aligned}
& V_{\mathrm{TH}}=V_{\text {sat }}^{+}\left(1-\frac{R_{F}}{R_{L}}\right)+v_{i}(t) \frac{R_{F}}{R_{S}}, \\
& V_{\mathrm{TL}}=V_{\text {sat }}^{-}\left(1-\frac{R_{F}}{R_{L}}\right)+v_{i}(t) \frac{R_{F}}{R_{S}} .
\end{aligned}
$$

These values of $V_{\mathrm{TH}}$ and $V_{\mathrm{TL}}$ result in $T_{\mathrm{on}}$ and $T_{\text {off }}$ as follows:

$$
\begin{aligned}
& T_{\text {on }}=R_{F} C \ln \frac{\left[2 R_{L} / R_{F}-1\right]-v_{i}(t) R_{L} / V_{\text {sat }}^{+} R_{S}}{\left[1-v_{i}(t) R_{L} / V_{\text {sat }}^{+} R_{S}\right]}, \\
& T_{\text {off }}=R_{F} C \ln \frac{\left[2 R_{L} / R_{F}-1\right]-v_{i}(t) R_{L} / V_{\text {sat }}^{-} R_{S}}{\left[1-v_{i}(t) R_{L} / V_{\text {sat }}^{-} R_{S}\right]} .
\end{aligned}
$$

The overall period of the modulated output is given by

$$
T=T_{\text {on }}+T_{\text {off }}
$$

and the duty factor $(D)$ can be computed as

$$
D=\frac{T_{\text {on }}}{T} \times 100 \% \text {. }
$$

Equation (4) shows that the duty cycle of the output can be controlled with the help of modulating signal $v_{i}(t)$.

\section{Realizing an OTRA and Nonideality Analysis}

For the proposed PWM circuit, the OTRA was realized using AD844 CFOA IC as shown in Figure 5 [12]. The equivalent circuit of Figure 5 for nonideal analysis [13] is presented in Figure 6. The CFOAs have been replaced with current conveyors having finite input resistances $\left(R_{X}\right)$ and finite 


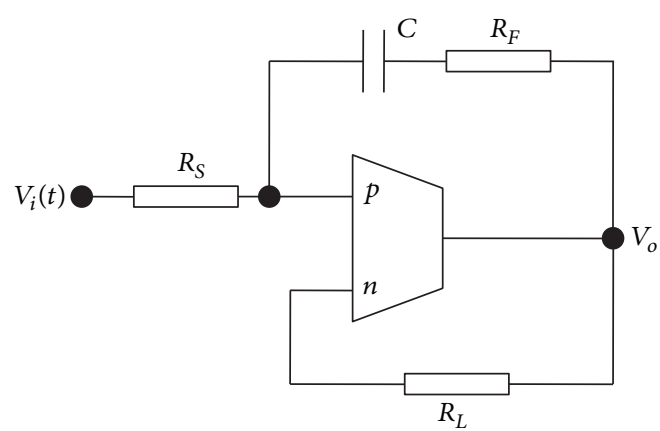

FIGURE 3: The OTRA PWM circuit.

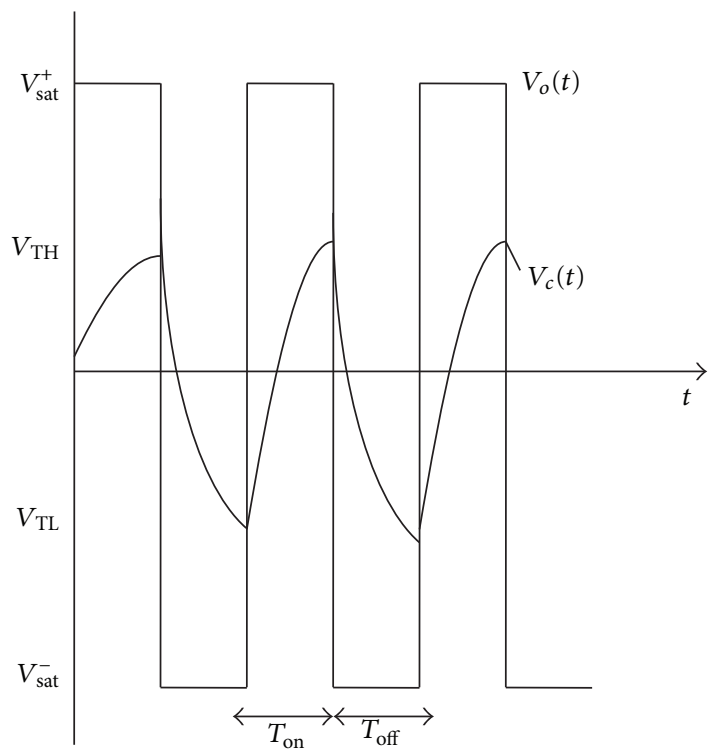

FIGURE 4: Output of the square wave generator of Figure 3.

resistance at its $Z$ terminal $\left(R_{Z}\right)$. Ideally, the input resistance at the $X$ terminal is zero and is infinite at the $Z$ terminal. For the AD844 CFOA, the input resistance $R_{X}$ is around $50 \Omega$, and $R_{Z}$ is around $3 \mathrm{M} \Omega$ [14].

From Figure 6, various currents can be calculated as follows:

$$
\begin{gathered}
I_{Z 1}=I_{+}, \\
I_{D}=I_{Z 1}\left(\frac{R_{Z}}{R_{X}+R_{Z}}\right), \\
I_{X 2}=I_{-}-I_{D}, \\
I_{Z 2}=I_{X 2} .
\end{gathered}
$$

Ideally, $I_{D}$ should be equal to $I_{Z 1}$, which can be approximated only if $R_{Z} \gg R_{X}$, which is true for $\mathrm{AD} 844$. Also,

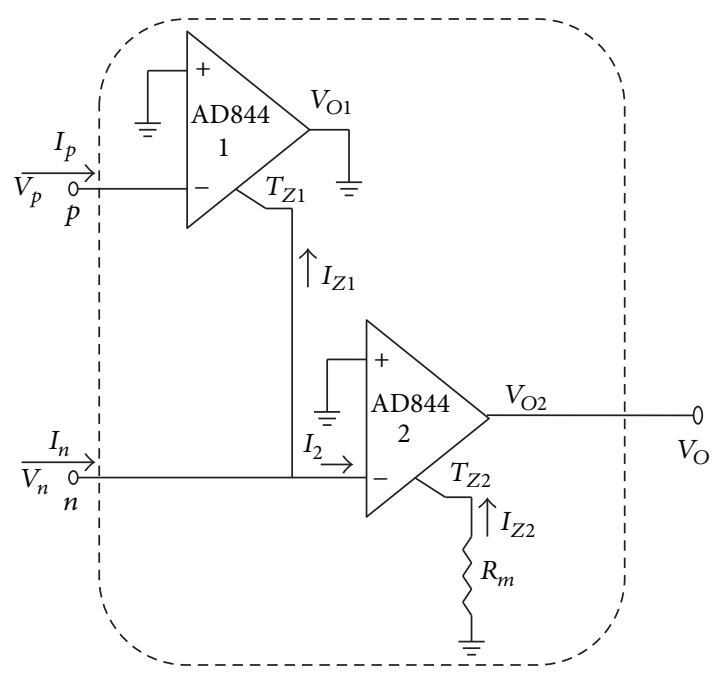

Figure 5: OTRA constructed with AD844.

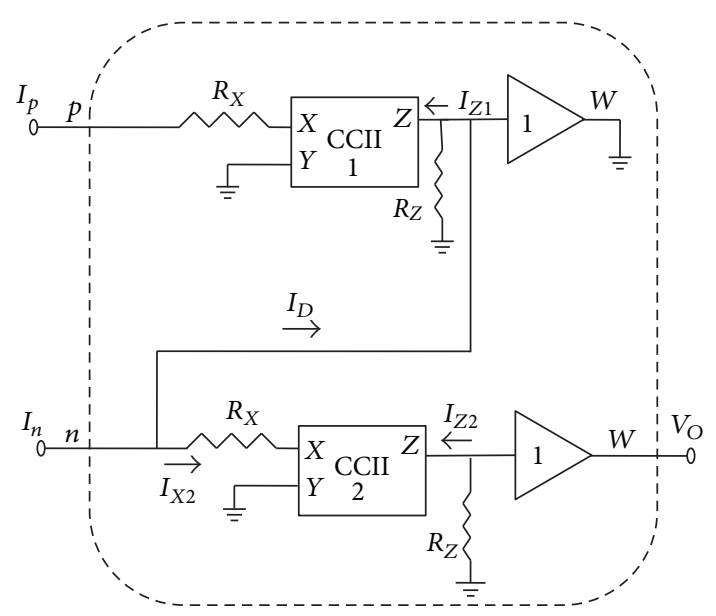

FIGURE 6: Equivalent circuit of OTRA constructed with AD844.

the approximation that the input terminals are virtually grounded will be true only if the external resistance at the input terminal of the OTRA is much larger than $R_{X}$. If these two conditions are satisfied, the OTRA constructed with AD844 closely approximates an ideal OTRA.

From (7), the output voltage $V_{O}$, taking into account the previously mentioned approximations, can be calculated as

$$
V_{O}=\left(I_{+}-I_{-}\right) R_{Z}
$$

where $R_{Z}$ is the transimpedance gain of the OTRA.

If in PWM circuit shown in Figure 3 the equivalent circuit of OTRA constructed with AD844 is used, then the threshold limits of the output get modified to

$$
\begin{aligned}
& V_{\mathrm{TH}}=V_{\text {sat }}^{+}\left(1-\frac{R_{F}+R_{x}}{R_{L}+R_{X} / / R_{Z}}\right)+v_{i}(t) \frac{R_{F}+R_{x}}{R_{S}+R_{x}}, \\
& V_{\mathrm{TL}}=V_{\text {sat }}^{-}\left(1-\frac{R_{F}+R_{x}}{R_{L}+R_{X} / / R_{Z}}\right)+v_{i}(t) \frac{R_{F}+R_{x}}{R_{S}+R_{x}} .
\end{aligned}
$$




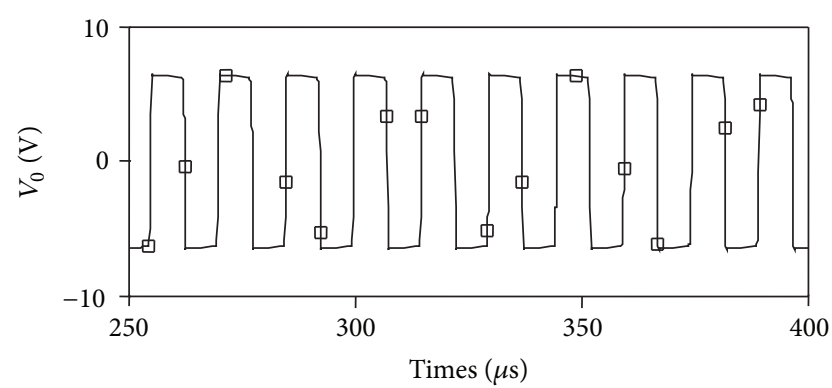

FigURE 7: PWM output without modulating signal.

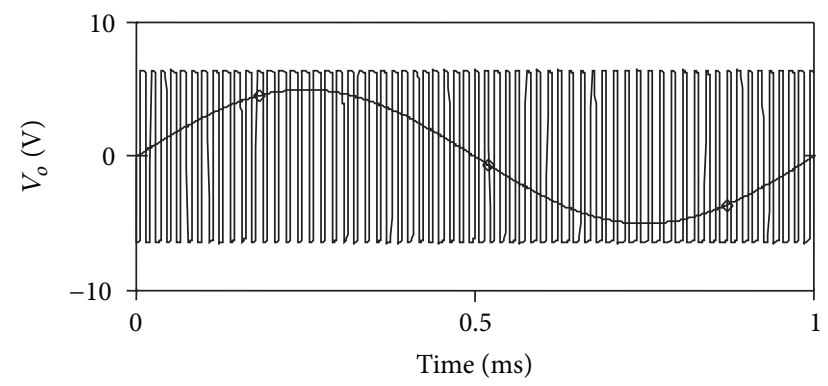

(a) Modulating signal and PWM output signal

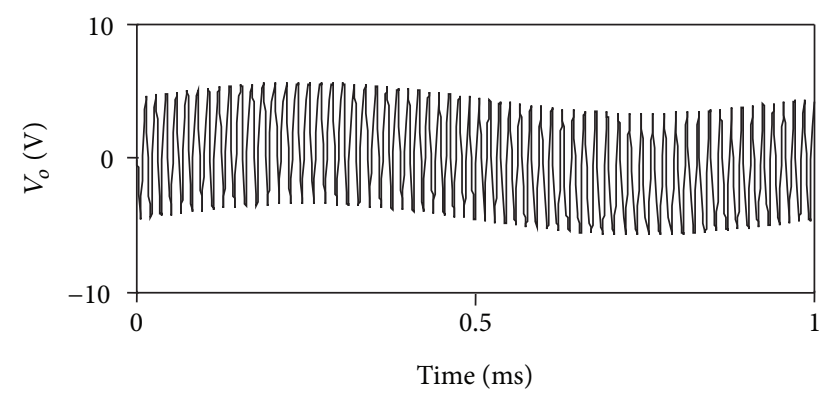

(b) Summed up modulating and carrier signals

FIGURE 8: PWM output for $5 \mathrm{~V}, 1 \mathrm{KHz}$ sinusoidal modulating signal.

The external resistance at the input of the OTRA should be much larger than $R_{X}$ so that the feedback current can be absorbed into the input terminals. Since $R_{L}, R_{F}, R_{S}$, and $R_{Z} \gg R_{X}$, Equations (9) and (10) reduce to (2) and (3), respectively.

\section{Simulation and Experimental Results}

The proposed circuit is simulated using PSPICE. OTRA is realized using current feedback operational amplifiers (CFOAs) IC AD844 as shown in Figure 5. Macro model of AD844 is used for simulations. Figure 7 shows the output voltage of the PWM when modulating signal is not applied, for $R_{F}=20 \mathrm{~K} \Omega, R_{L}=70 \mathrm{~K} \Omega, R_{S}=80 \mathrm{~K} \Omega$, and $\mathrm{C}=$ $200 \mathrm{pF}$, and corresponds to a $50 \%$ duty cycle. The observed output frequency is $66.7 \mathrm{KHz}$ as against the calculated value of $69.7 \mathrm{KHz}$.

Simulated output of pulse width modulator, with same component values as used in Figure 7, is shown in Figure

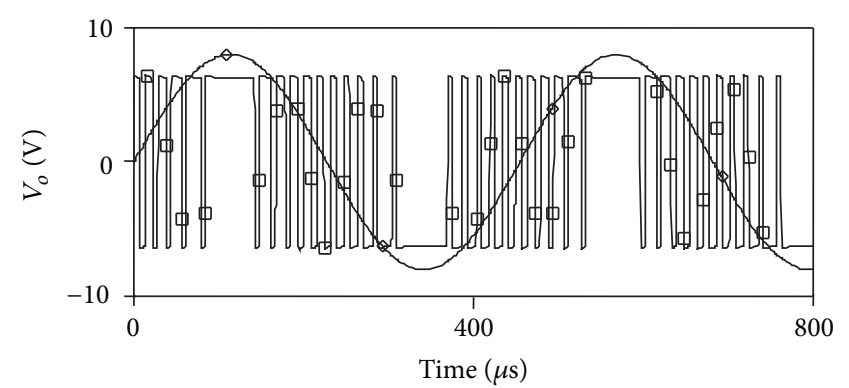

(a) Modulating signal and PWM output signal

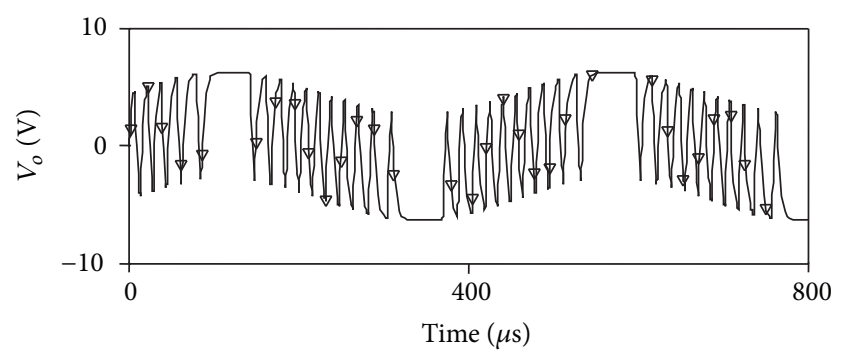

(b) Summed up modulating and carrier signals

FIGURE 9: PWM output of for $8 \mathrm{~V}, 2.2 \mathrm{KHz}$ modulating signal.

8 for a $5 \mathrm{~V}, 1 \mathrm{KHz}$ sinusoidal modulating signal. Figure 8(a) shows the modulating signal and the PWM output, and summation of modulating and carrier wave is depicted in Figure $8(\mathrm{~b})$. Figures 9 and 10 show the output of pulse width modulator for an $8 \mathrm{~V}, 2.2 \mathrm{KHz}$ modulating signal and $5 \mathrm{~V}, 40 \mathrm{KHz}$ modulating signal, respectively, for same set of component values. Frequency spectrum of the pulse width modulator for $8 \mathrm{~V}, 2.2 \mathrm{KHz}$ modulating signal is shown in Figure 11 which consists of modulating component and carrier signal. Thus, the modulating signal can be recovered with the help of appropriate low pass filter.

Figure 12 shows the variation of theoretically computed and simulated $\%$ duty factor of the modulator with the applied input signals. This shows that the two results closely match with each other.

The functionality of the proposed pulse width modulator circuits is verified through hardware as well.

The commercial IC AD844AN is used to implement an OTRA. Supply voltages used are \pm 5 V. Figure 13 shows typical experimental results of the circuit for two different modulating signals. Figure 13(a) depicts output for component values $R_{F}=20 \mathrm{~K} \Omega, R_{L}=70 \mathrm{~K} \Omega, R_{S}=80 \mathrm{~K} \Omega$ and $\mathrm{C}=200 \mathrm{pF}$ and for an $8 \mathrm{~V}, 2.2 \mathrm{KHz}$ modulating signal. Another screen shot of oscilloscope is shown in Figure 13(b) for a high-frequency modulating signal of $40 \mathrm{KHz}$ with $5 \mathrm{~V}$ amplitude. These experimental results are in close agreement to simulated results. 
TABLE 1: Comparison between the proposed and the previously reported works.

\begin{tabular}{|c|c|c|c|c|}
\hline References & no. of active components & No. of passive components & Carrier signal type & $\begin{array}{l}\text { Electronic } \\
\text { tunability }\end{array}$ \\
\hline$[4]$ & Single CC-II, two op-amps & Single capacitor, three resistors & Triangular & No \\
\hline$[6]$ & Single op-amp & Single capacitor, three resistors & Exponential & No \\
\hline \multirow{2}{*}{ [7] } & (i) Two OTAs, an inverter, and a MOS switch & (i) Single capacitor, single resistor & (i) Sawtooth & Yes \\
\hline & (ii) Four OTAs and an inverter & (ii) Single capacitor, single resistor & (ii) Triangular & Yes \\
\hline [8] & Three OTAs & Single capacitor, two resistors & Triangular & Yes \\
\hline Proposed & Single OTRA & Single capacitor, three resistors & Exponential & No \\
\hline
\end{tabular}

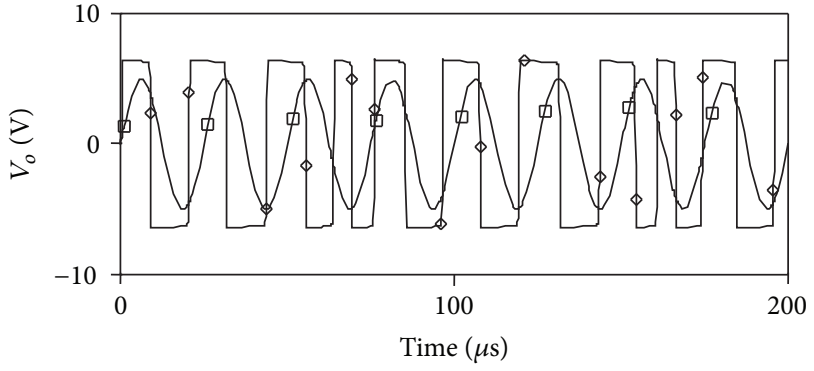

(a) Modulating signal and PWM output signal

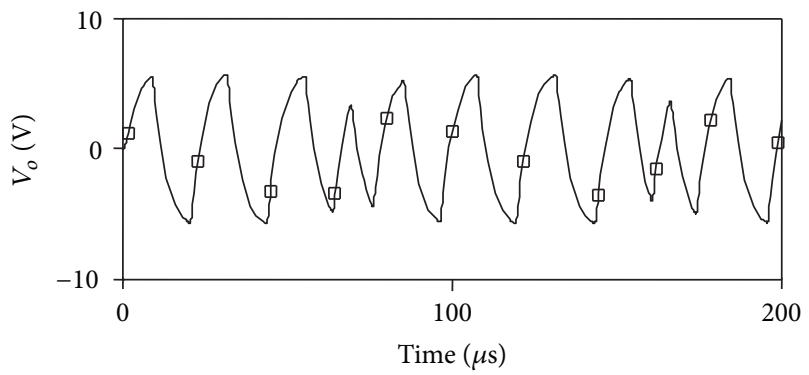

(b) Summed up modulating and carrier signals

FIGURE 10: PWM output for $5 \mathrm{~V}, 40 \mathrm{KHz}$ modulating signal.

\section{Comparison}

In this section, a comparison of the proposed work with the previously reported analog PWM circuits $[4,6-8]$ is presented, which are all based on the concept of comparing a modulating signal with a reference sawtooth or triangular waveform to generate PWM signal. Table 1 shows the detailed comparison. The study of Table 1 reveals that topologies presented in $[4,7,8]$ use more number of active components as compared to the proposed work. The proposed circuit is simpler as compared to the topologies of $[4,7,8]$, since it uses the exponential voltage waveform across the capacitor of the square wave generator as carrier wave and, hence, does not require additional circuitry needed for triangular/sawtooth waves. Though exponential carrier wave being a nonlinear signal yields

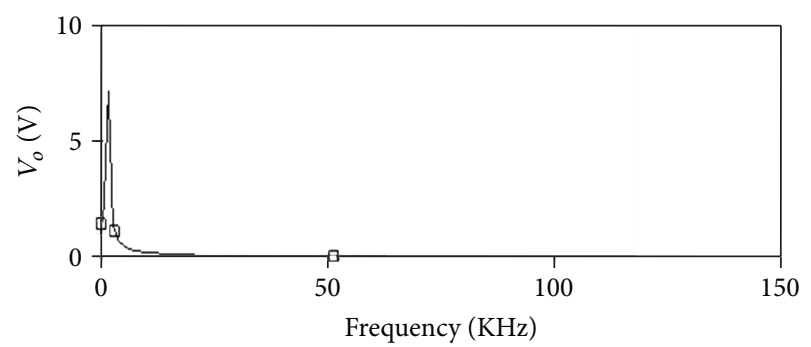

(a) Frequency spectrum of modulating signal

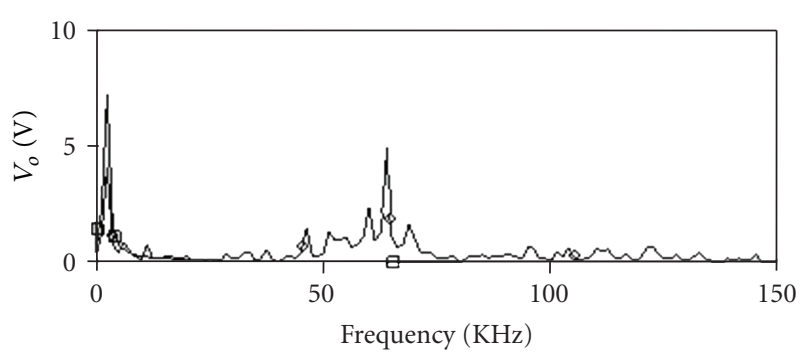

(b) Frequency spectrum of modulated signal

FIgURE 11: Frequency spectrum of the PWM.

relatively inaccurate PWM signal at lower carrier frequencies as compared to the triangular/sawtooth wave, yet one can trade off accuracy for simplicity depending upon the application.

The PWM topology of [6], which is a classical opampbased design, uses same number of active and passive components as in proposed circuit. However, the opampbased circuits show limited high-frequency performance due to lower slew rate and constant gain-bandwidth product of the op-amps. Additionally, the input terminals of OTRA being virtually grounded, the proposed circuit is free from parasitic effects.

\section{Conclusion}

In this paper, single OTRA-based PWM generator, a nonlinear application of OTRA, is proposed. This circuit can be used for speed control of DC motors and for DC-DC converters. 


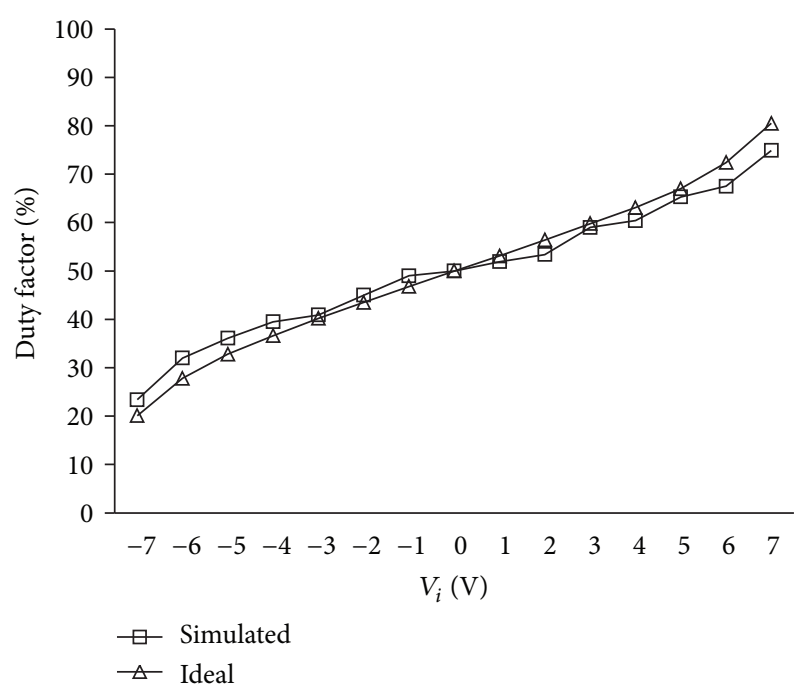

FIGURE 12: Variation of duty factor with applied input signal.

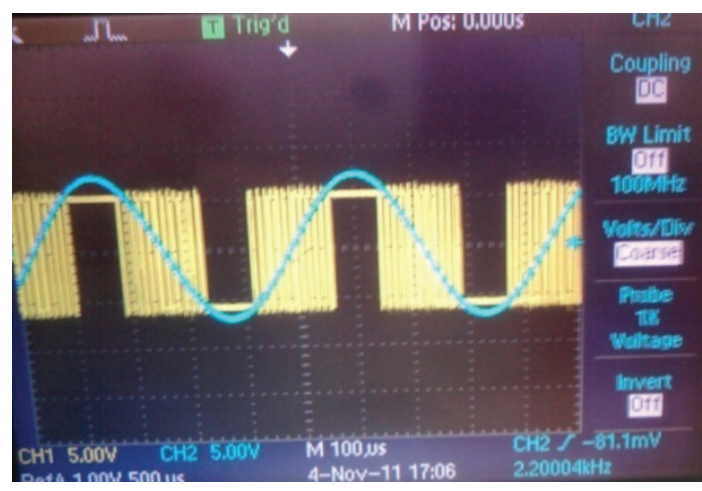

(a) PWM output for an $8 \mathrm{~V}, 2.2 \mathrm{KHz}$ modulating signal

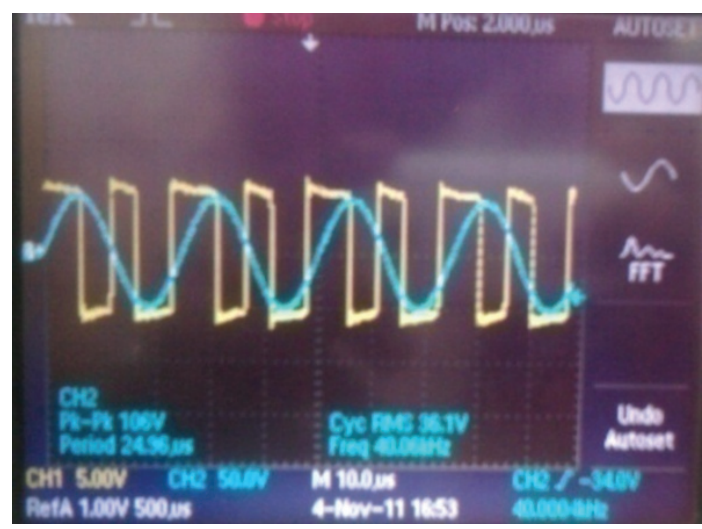

(b) PWM output for a $5 \mathrm{~V}, 40 \mathrm{KHz}$ modulating signal

FIGURE 13: Experimental results of the proposed PWM.

PSPICE simulation results using AD844 macromodel and experimental results have been included for verification of the theoretical propositions which are found in close agreement.

\section{References}

[1] W. Mathis, "Nonlinear systems and communications systems," in Proceedings of the International Conference on Telecommunication in Modern Satellite, Cable and Broadcasting Services (TELSIKS '01), vol. 1, pp. 293-296, September 2001.

[2] M. S. Roden, Analog and Digital Communication Systems, Prentice-Hall, Englewood Cliffs, NJ, USA, 4th edition, 1996.

[3] D. Maksimovic and S. Cuk, "A unified analysis of PWM converters in discontinuous modes," IEEE Transactions on Power Electronics, vol. 6, pp. 476-490, 1991.

[4] M. Siripruchyanun, P. Wardkein, and W. Sangpisit, "Simple pulse width modulator using current conveyor," in Proceedings of the TENCON '00), vol. 1, pp. 452-457, September 2000.

[5] A. S. Sedra and K. C. Smith, Microelectronic Circuits, Oxford University, 2004.

[6] APEX Microtechnology, "PWM Basics, Pulse Width Modulator Amplifier," Application Note 30.

[7] H. Kim, H. J. Kim, and W. S. Chung, "Pulsewidth modulation circuits using CMOS OTAs," IEEE Transactions on Circuits and Systems I, vol. 54, no. 9, pp. 1869-1878, 2007.

[8] M. Siripruchyanun and P. Wardkein, "A fully independently adjustable, integrable simple current controlled oscillator and derivative PWM signal generator," IEICE Transactions on Fundamentals of Electronics, Communications and Computer Sciences, vol. 86, no. 12, pp. 3119-3126, 2003.

[9] A. Djemouai, M. Sawan, and M. Slamai, "New CMOS integrated pulse width modulator for voltage conversion applications," in Proceedings of the IEEE International Conference on Electronics, Circuits and Systems (ICECS '00), vol. 1, pp. 116-119, December 2000.

[10] J.-J. Chen, H.-Y. Lin, Y.-T. Lin, and W.-Y. Chung, "Integrated pulse-width-modulation circuit using CMOS processes," in Proceedings of the Power Electronics Specialists (IEEE PESC '04), pp. 1356-1358, June 2004.

[11] Y. Zheng and C. E. Saavedra, "Pulse width modulator using a phase-locked loop variable phase shifter," in IEEE International Symposium on Circuits and Systems (ISCAS '05), pp. 3639-3642, May 2005.

[12] C. L. Hou, H. C. Chien, and Y. K. Lo, "Squarewave generators employing OTRAs," IEE Proceedings Circuits, Devices and Systems, vol. 152, no. 6, pp. 718-722, 2005.

[13] Y. K. Lo, H. C. Chien, and H. J. Chiu, "Switch-controllable OTRA-based bistable multivibrators," IET Circuits, Devices and Systems, vol. 2, no. 4, pp. 373-382, 2008.

[14] AD 844 Datasheet, Analog Devices Inc. 

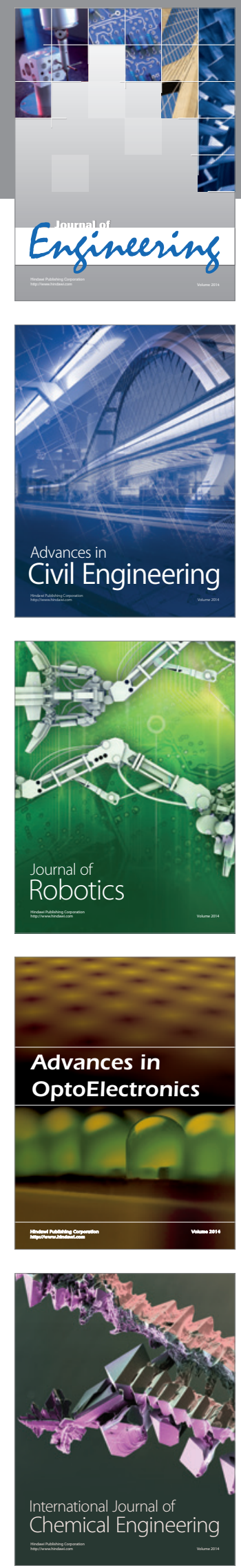

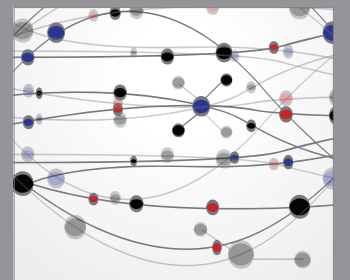

The Scientific World Journal
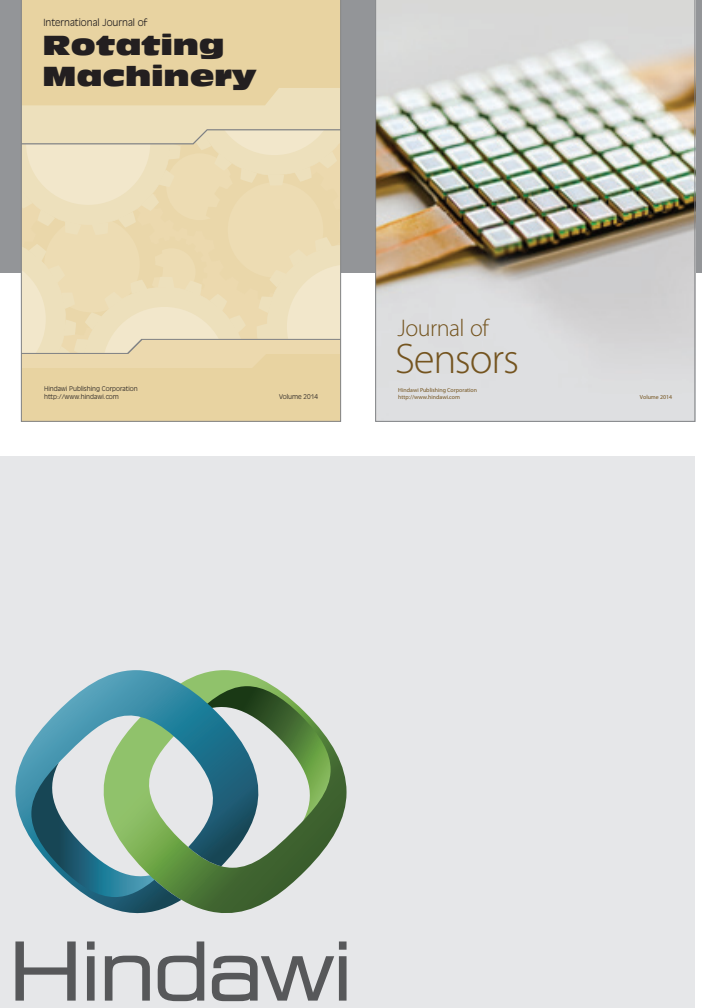

Submit your manuscripts at http://www.hindawi.com
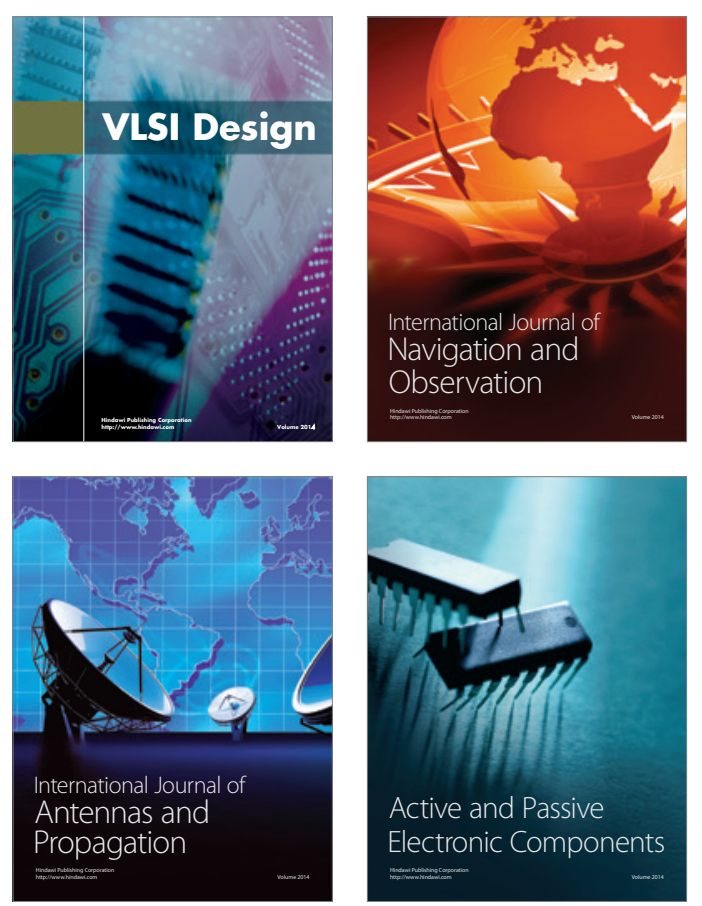
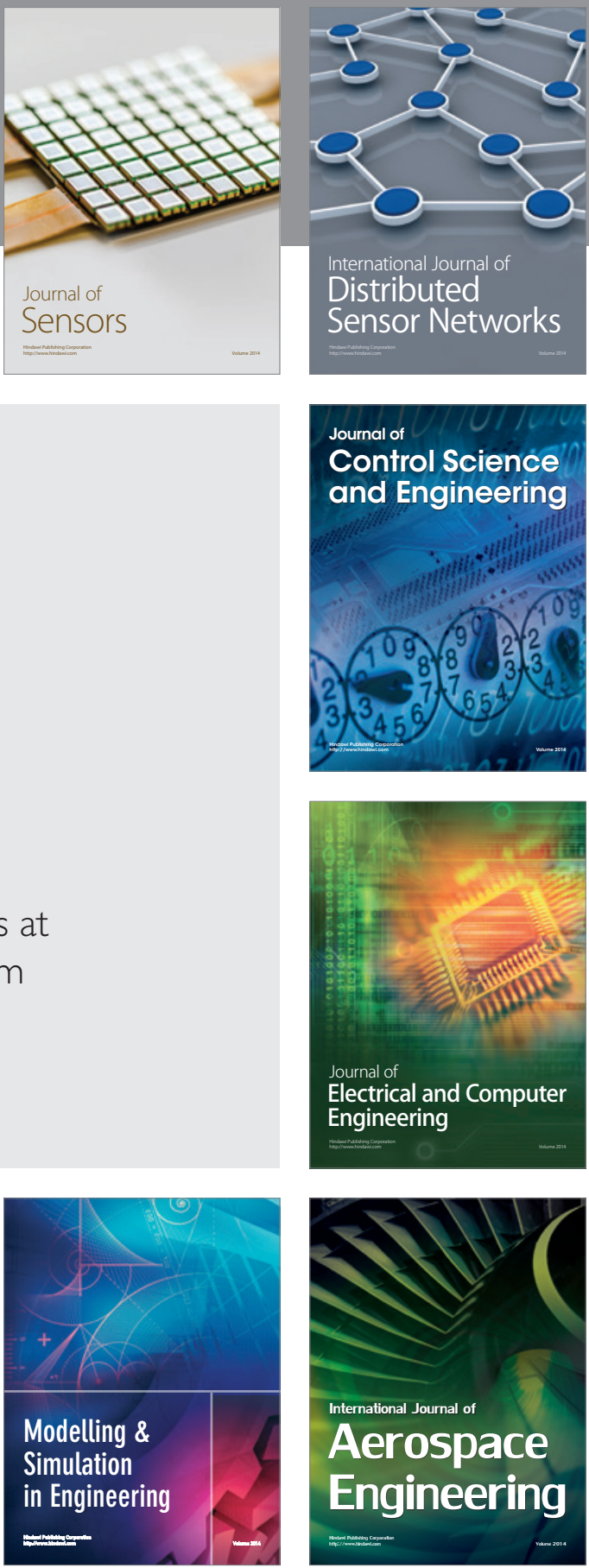

Journal of

Control Science

and Engineering
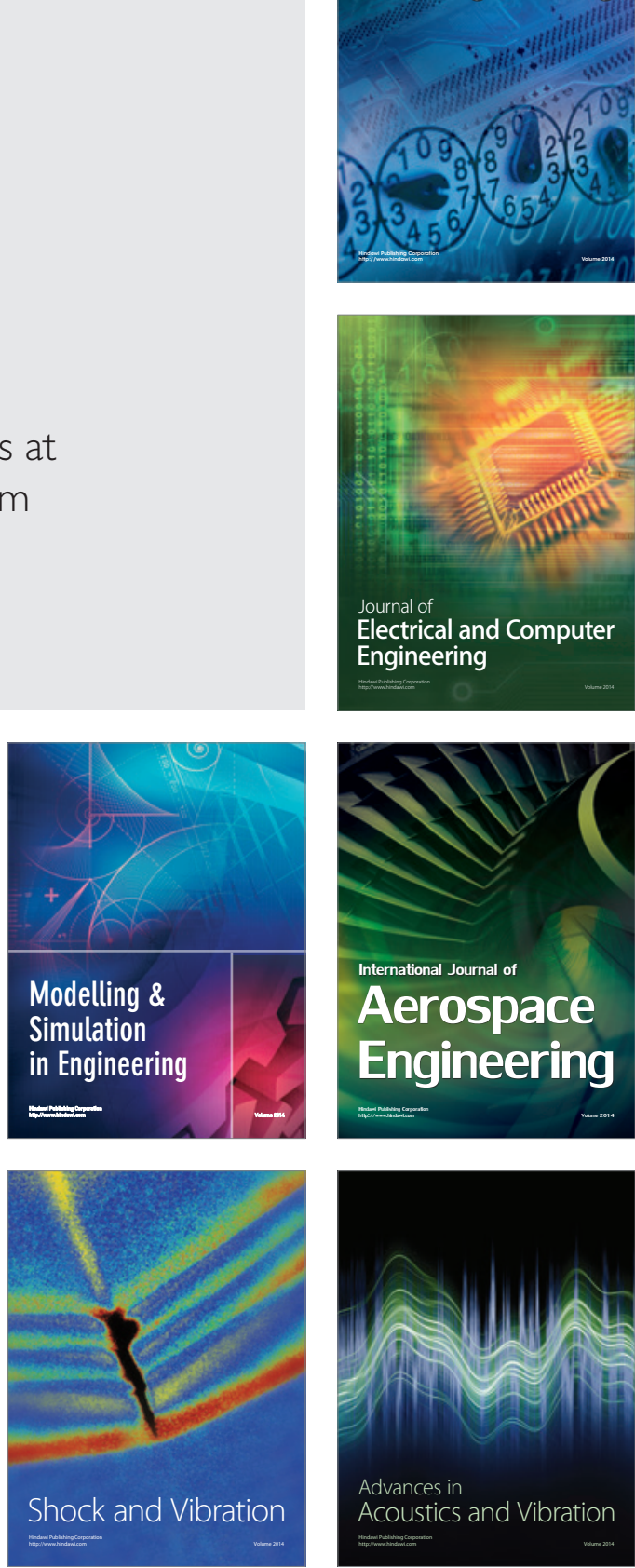